\title{
REMOVABLE OR FIXED??? FORCE IS IN THE HANDS OF ORTHODONTIST NOT APPLIANCE
}

\author{
Garima Gupta ${ }^{1}$, Sachin Goyal ${ }^{2}$ \\ ${ }^{1}$ Reader, Deptt. of Orthodontics and Dentofacial Orthopedics, K.D. Dental College and Hospital, Mathura. \\ ${ }^{2}$ Reader, Deptt. of Periodontology, Bhojia Dental College \&Hospital, Budh, Baddi, Himachal Pradesh.
}

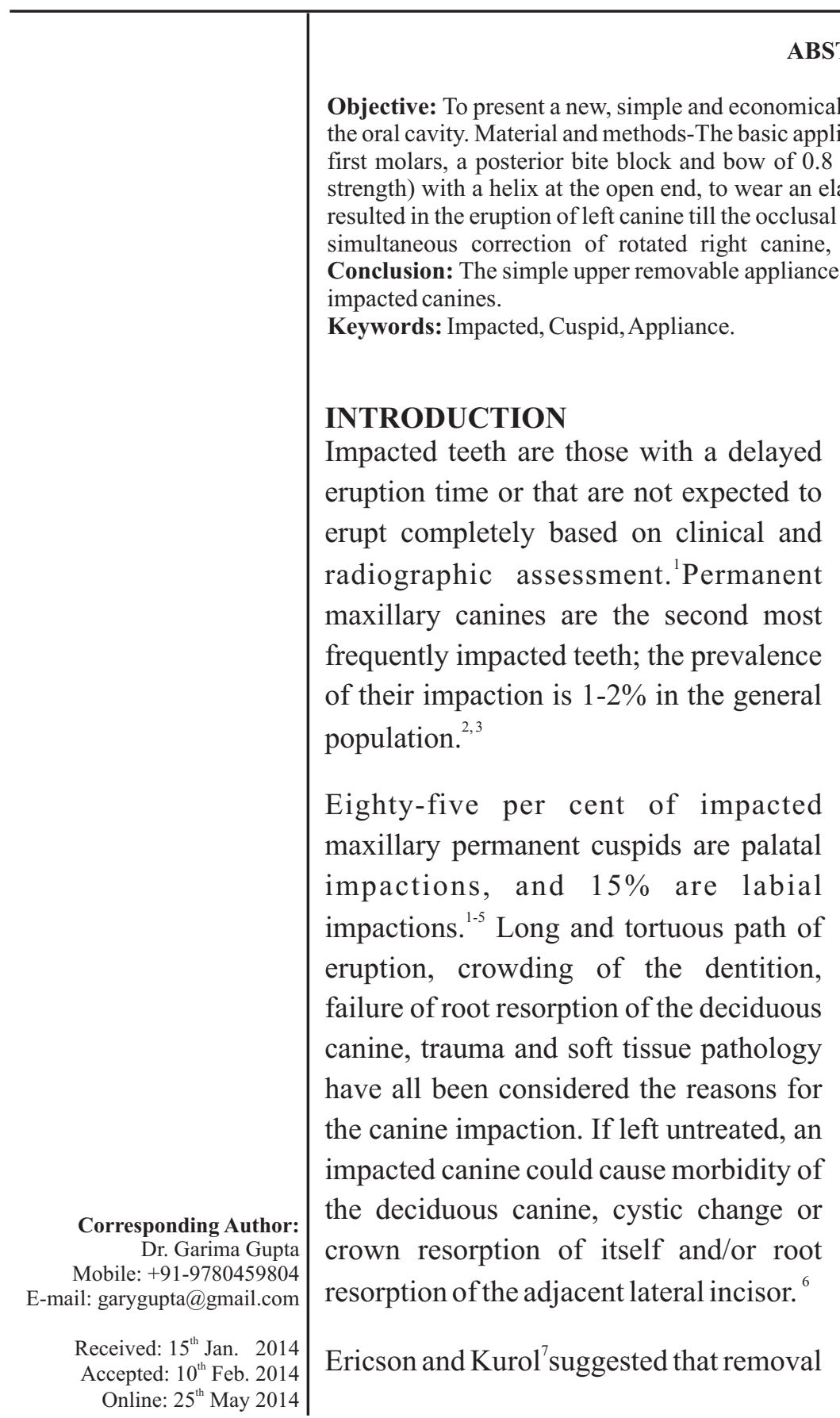

of the deciduous canine before the age of 11 years will normalize the position of the ectopically erupting permanent canine in $91 \%$ of cases if the canine crown is distal to the midline of the lateral incisor.

Methods of diagnosis that may allow for early detection and prevention should include a family history, visual and tactile clinical examinations by the age of ${ }^{8-10}$ years and a thorough radiographic assessment.

The conventional treatment approach of an impacted canine includes surgical and fixed orthodontic therapy wherein crown of the canine is exposed surgically, followed by bonding an attachment onto the crown surface. The attachment is then ligated to the arch wire in the mouth. Attention, however, has been focused on the long-term health of the gingiva and the unacceptable length of the clinical crown often seen after this procedure. Although it seems like the ideal approach towards 
management of an impacted canine, but many a times it's not a viable option due to patients economic and time constraints. Rare earth magnets and lower removable appliances have been reported for extrusion of impacted maxillary canines. ${ }^{8,9}$

This article will present a new, simple and economical method of bringing an impacted canine into the oral cavity. First a well-clasped upper removable appliance is shown to be an excellent base for using light elastics to move the impacted canine vertically and buccally. An attachment is bonded to the unerupted canine to provide a simple and flexible means of traction that ensures the canine erupts into a zone of attached mucosa with normal crown length. Followed by another removable appliance with a cantilever spring tied to the molar tube soldered to the Adam's clasp, which when engaged into the canine will bring the canine vertically down.

\section{MATERIALAND METHODS}

The appliances will be described in detail with the help of a treated case. The upper removable appliance for traction to impacted maxillary canines.

The basic appliance design incorporates Adams clasps, usually on both first molars, a posterior bite block and bow of $0.8 \mathrm{~mm}$ wire with a stainless steel tubing (to increase the strength) with a helix at the open end. A double cantilever spring added on the right side to simultaneously derotate and move the canine buccally as the patient presented with crossbite in relation to right canine (Figure 1).

\section{Case diagnosis and treatment planning}

14 year old patient visited the department with the chief complaint of unerupted upper left side tooth. On examination it was observed that upper left canine is clinically absent. Panoramic radiograph showed impacted upper left canine. Occlusal radiograph depicted palatally positioned canine. The impacted canine was positioned favourably and complicated biomechanics was not required. Also the right canine was in cross bite with the lower canine resulting in shift in the mandibular position in centric occlusion. Lower midline was shifted to right by $2 \mathrm{~mm}$ (Figure 2).
Surgical exposure was carried out. The bracket was then bonded on the exposed surface. Before bonding the bracket, a ligature wire was tied to it and twisted to form a long pig tail tie with a hook made at its free end. This extension was to dangle down into the oral cavity through the flap that is replaced to cover the tooth with bracket bonded to its crown (Figure 3). By means of this wire, the tooth was pulled to its destined position in the arch. Patient was advised to wear $1 / 8$ inch elastic from the ligature hook from the tooth to the helix on the appliance (Figure 1).

\section{Treatment progress}

Patient was monitored at regular intervals with first recall after one week of appliance placement to monitor progress and reinforce compliance with elastics and removable appliance. The double cantilever spring was activated at this appointment. Four months into removable therapy patient showed eruption of canine in its proper position through attached mucosa. As the distance between the ligature hook and the eyelet on the appliance was reduced, the elastic force decreased significantly. Also, the vector of force required, shifted from being buccal and vertical to more vertical. Thus, a new removable appliance, with a cantilever spring tied to the molar tube soldered to the Adam's clasp was fabricated (Figure 4). A pre-activation bend in the occlusal direction was placed near the molar tube. An attachment was bonded on the buccal surface of the canine, to engage the spring (Figure 4).

\section{RESULTS}

The left canine erupted till the level of occlusal table and simultaneously the right canine derotated and moved buccally resolving the cross bite. This led to the correction of mandibular shift and the lower midline coincided with the upper midline. Total treatment time was 6 months. Post treatment photographs show erupted left canine in occlusion, normally occluding right canine, coinciding midlines and no mandibular shift (Figure 5). 


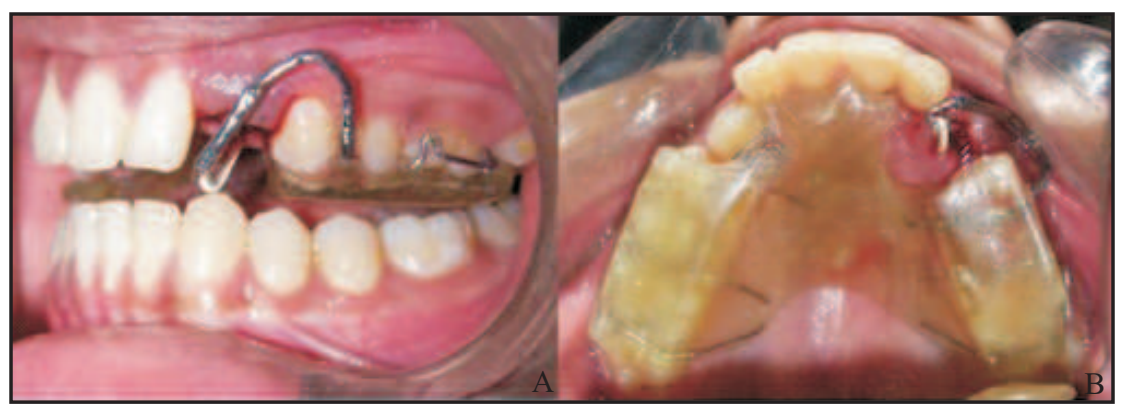

Figure 1 (A, B) : Removable Appliance in place

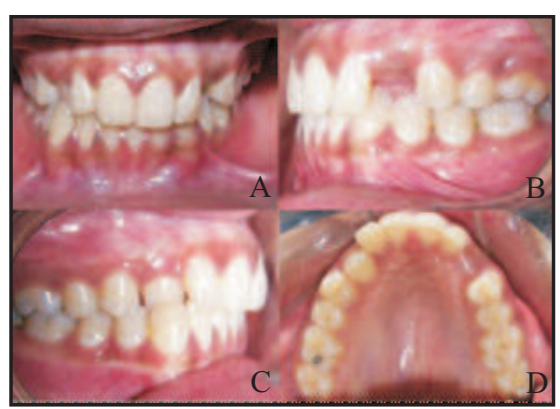

Figure 2(A, B, C, D): Pre-treatment

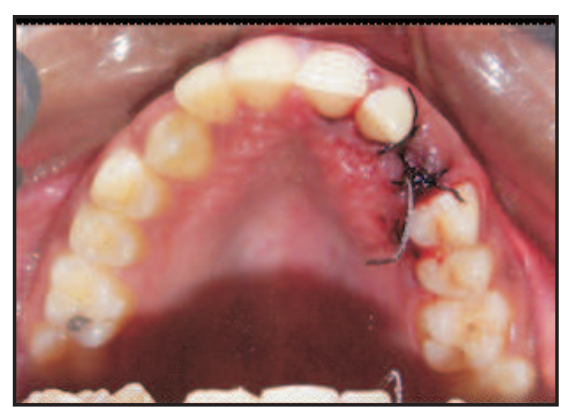

Figure 3: Surgical exposure of impacted canine with pigtail ligature dangling from bonded attachment

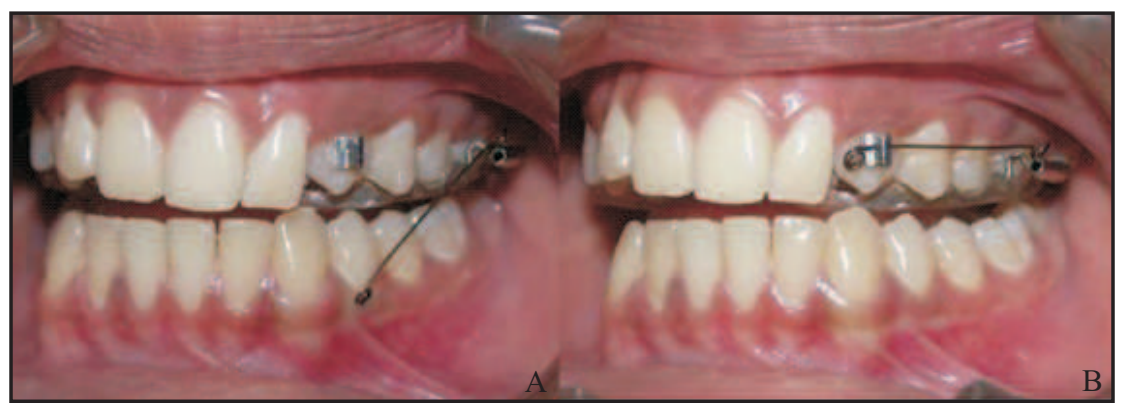

Figure 4(A, B): Appliance with cantilever spring in place

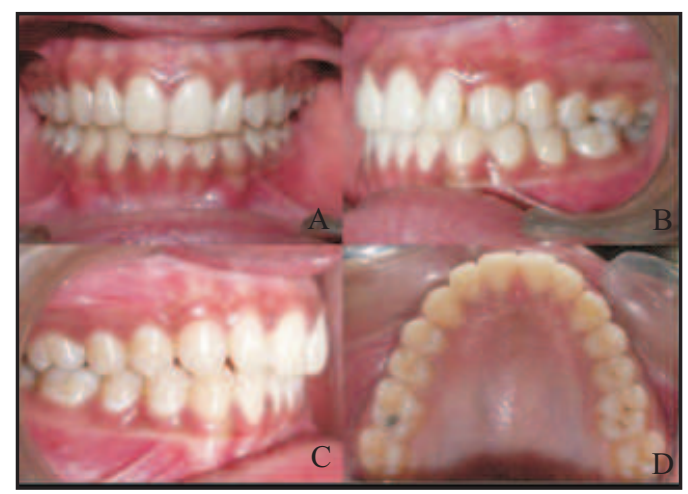

Figure 5(A, B, C, D): Post-treatment 


\section{DISCUSSION}

Impaction of the maxillary canine is a problem frequently encountered in orthodontic practice. The complexity of diagnosis and treatment plan taxes the orthodontist's and surgeon's intelligence. Indeed, there are several modalities in treating impacted maxillary canines dictated by several parameters. Routinely fixed orthodontic treatment is the treatment of choice as the biomechanics involved are complicated and require a three dimensional control over canine. But many a times such complex treatment is not warranted. Proper diagnosis and treatment planning is the key to the success of any appliance therapy. A new simplified appliance is shown to produce the desired tooth movement on the foundation of sound diagnosis and treatment planning.

Once the force vector required moving the canine is established, force delivery system is provided with a removable appliance incorporating a posterior bite block, cantilever arm with tubing and elastic component. Force delivered was in vertical and buccal direction. Direction and magnitude of force can be altered by changing the length of cantilever arm.

The overwhelming advantage of this appliance is the control over the force and the direction of applied traction that always has an inferiorly directed vector. The advantage of using an upper removable appliance is that the resistance to the applied force tends to seat the appliance, whereas with a lower removable appliance, it will obviously tend to dislodge it. One distinct advantage is that the appliance can be modified and various active components can be added to suit a particular clinical situation. Simultaneously the force direction and magnitude can be altered according to the position of the concerned tooth and biomechanics required. The appliance can also be used for palatally erupted canines to get them into arch if space available is adequate. Being removable, the appliance is patient friendly, easy to maintain oral hygiene and requires minimal chair side time.

Probable limitation of appliance is when space required to align the impacted tooth is inadequate.in such case the biomechanics involved are complex and thus warrants fixed orthodontic therapy. Potential problem is as with any removable appliance, i.e. dependence on patient cooperation, which can be managed with guidance and reinforcement.

\section{CONCLUSION}

The simple upper removable appliance can be used as an excellent method of treating favourably impacted palatal canines.

\section{REFERENCES}

1. Thilander B, Jakobsson SO. Local factors in impaction of maxillary canines. Acta Odontol Scand 1968; 26:145-68.

2. Rayne J. The unerupted maxillary canine. Dent Pract Dent Rec 1969; 19:194-204.

3. Bass TB. Observations on the misplaced upper canine tooth. Dent Pract Dent Rec 1967; 18:25-33.

4. Hitchin AD. The impacted maxillary canine. Br Dent J. 1956;100:1-14.

5. Ericson S, Kurol J. Radiographic examination of ectopically erupting maxillary canines. Am J Orthod Dentofac Orthop. 1987;91:483-492.

6. Becker A: Palatally impacted canines. In The orthodontic treatmentof impacted teeth Second edition. Andover, Hampshire: Thomson PublishingServices; 2007:93-142.

7. Ericson S, Kurol J. Early treatment of palatally erupting maxillary canines by extraction of the primary canines. Eur J Orthod. 1988;10:283-295.

8. Larry CF Li1, Ricky WK Wongand Nigel M King. Orthodontic traction of impacted canine using magnet: a caseReport.Cases Journal 2008; 1:382.

9. Harry S. Orton, M.Thérèse Garvey Michael H. Pearson. Extrusion of the ectopic maxillary canine using a lower removable appliance. Am J Orthod Dentofac Orthop 1995; 107:349-59. 